\title{
Linfoma marginal del bazo asociado a acidosis láctica tipo B. Caso clínico
}

${ }^{1}$ Servicio de Medicina Interna, Sección de Nefrología, Hospital Naval A. Nef, Viña del Mar, Chile. ${ }^{2}$ Departamento de Medicina, Escuela de Medicina, Universidad de Valparaíso, Valparaíso, Chile. ${ }^{3}$ Servicio de Medicina Interna, Sección de Hematología, Hospital Naval A. Nef, Viña del Mar, Chile.

Recibido el 24 de junio de 2011, aceptado el 13 de septiembre de 2011.

Correspondencia a a: Dr. Jorge Vega Stieb 5 Norte 1035, Viña del Mar, Chile.

Fono: 56-32-2974237

Fax: 56-32-2970050

E-mail: jvegastieb@gmail.

\author{
JORGE VEGA ${ }^{1,2}$, MARÍA DE LOS ÁNGELES RODRÍGUEZ ${ }^{3}$, \\ ARMANDO PEÑA ${ }^{3}$, ALEJANDRO VÁSQUEZ ${ }^{3}$
}

\section{Type B lactic acidosis associated with marginal lymphoma of the spleen. Report of one case}

Lactic acidosis in the absence of hypoxia or tissue hypoperfusion (type B) is very rare and is associated with the use of some drugs or malignancy. We report a 79-year-old woman, with a marginal non-Hodgkin's lymphoma of the spleen that was subjected to a splenectomy one year ago. She presented with unexplained tachypnea associated with pancytopenia and elevation of IgM to 10 times over the higher normal limit. Laboratory tests showed the presence of metabolic acidosis and high lactic acid levels in the absence of infection, tissue hypoxia or hypoperfusion. She was treated with sodium bicarbonate and steroids without obtaining a reduction in lactate levels. Twelve days after admission, a single dose of Rituximab quickly normalized lactate concentrations and platelet count. After the fourth dose of Rituximab, pancytopenia disappeared and IgM fell to 25\% of its baseline concentration.

(Rev Med Chile 2012; 140: 236-242).

Key words: Acidosis, lactic; Antibodies, monoclonal, murine-derived; Lymphoma non-Hodgkin; Rituximab.
L a acidosis láctica (ACL) es un trastorno frecuente en pacientes hospitalizados, $\checkmark$ particularmente en pacientes sépticos o en shock. La hipoxia e hipoperfusión tisular inducen a las células a metabolizar la glucosa en forma anaeróbica, originando un aumento en la producción de lactato ${ }^{1}$. A este tipo de ACL se lo ha llamado tipo A. Cuando no existe un impedimento aparente en la oxigenación tisular se la denomina ACL tipo B (ACLB). Esta última es infrecuente y está relacionada con medicamentos (biguanidas, antiretrovirales), alcoholismo, déficit enzimáticos hereditarios y algunas neoplasias sólidas o hematológicas con gran masa tumoral ${ }^{1-4}$.

Comunicamos el caso de una anciana que a raíz de una disnea inexplicada se descubrió la existencia de una ACLB asociada a un linfoma no Hodgkin (LNH) marginal esplénico. Pensamos que la lectura de este caso hará incorporar a los procesos linfoproliferativos en el diagnóstico diferencial de una acidosis láctica de origen no precisado.

\section{Caso clínico}

Mujer de 79 años con antecedentes de apendicectomía y colecistectomía. En mayo de 2010 consultó por fatigabilidad, baja de peso con ingesta conservada, sudoración nocturna y equimosis espontáneas, que arrastraba desde hacía un año. Al examen físico había palidez, enflaquecimiento, esplenomegalia gigante sensible que alcanzaba la línea media y alcanzaba hasta la pelvis y una masa hipogástrica mal delimitada (Figura 1). No se palpaban adenopatías. Existía anemia, trombocitopenia y linfocitosis (algunos de apariencia "velluda") (Tabla 1). Fue hospitalizada con diagnóstico presuntivo de leucemia de células velludas (tricoleucemia). Un scanner mostró esplenomegalia gigante (286 $\mathrm{mm}$ de longitud), adenopatías en el hilio hepático y en el fondo de saco de Douglas. El mielograma mostró hiperplasia eritropoyética, hipoplasia granulocítica, mastocitosis (5-7\%), infiltración por linfocitos 


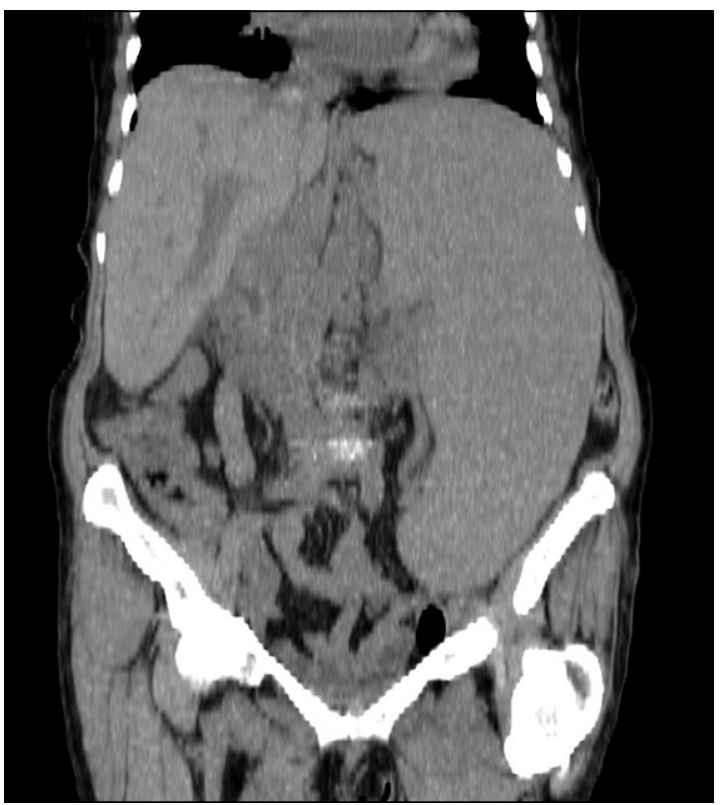

Figura 1. Imagen sagital del abdomen en tomografía computada durante la primera hospitalización. Se observa una esplenomegalia que alcanza hasta la cavidad pelviana y que es de un tamaño muy superior al hígado. En el hilio hepático existe un conglomerado de adenopatías.

maduros $(78 \%)$ y hemosiderina ausente. Una biopsia de médula ósea reveló hiperplasia de las 3 series y linfocitos pequeños $\mathrm{CD} 20(+)$ y CD3(-). No se efectuó inmunofenotipo porque la paciente no pudo solventar su costo. El patólogo concluyó que existía un síndrome linfoproliferativo de estirpe B.

Otras evaluaciones mostraron TSH elevada $(10,4$ uU.I./ml), alteraciones de la coagulación como: TTPK y tiempo de protrombina prolongado que no corregía con la adición de plasma fresco; factor VIII reducido y anticuerpos anticardiolipina IgM positivos (Tabla 1). La concentración de inmunoglobulina $\mathrm{M}$ fue 7 veces lo normal (monoclonal lambda en la inmunofijación en sangre) y la de beta 2 microglobulina estaba elevada. Los test de autoinmunidad fueron negativos con excepción del factor reumatoide. Fue esplenectomizada previa transfusión de eritrocitos y plaquetas, obteniéndose un bazo de 2.533 gramos cuya biopsia reveló acentuada hipercelularidad de la pulpa roja, tejido linfoide de la pulpa blanca atrófico constituido por linfocitos pequeños con diferenciación plasmocitoide. El $90 \%$ de las células linfoides fue CD20(+), $10 \% \mathrm{CD} 3(+)$ y $50 \% \mathrm{BCL} 2(+)$. Se con- cluyó que los hallazgos eran compatibles con una tricoleucemia. Fue dada de alta con prescripción de tiroxina y alopurinol. Posterior a ello la enferma no volvió a control.

Doce meses más tarde la paciente reapareció por disnea y náuseas. Al examen había palidez, taquicardia y polipnea notable. La presión arterial fue 120/70 mmHg, no había fiebre ni edema. La radiografía de tórax no mostró alteraciones que explicaran su disnea. La glicemia fue normal y había acidosis metabólica con anion gap y lactato elevados sin evidencias de hipoxemia, hipoperfusión tisular o infección sistémica (Tabla 2). El examen de orina fue normal y los hemocultivos y urocultivo negativos. Las enzimas cardiacas no fueron compatibles con un infarto al miocardio y salvo leve hiperbilirrubinemia, las pruebas hepáticas no mostraron evidencias de citolisis ni colestasia. El hemograma reveló pancitopenia, macrocitosis (VCM 108 $\mathrm{u}^{3}$ ), cuerpos de Howell Jolly y no se visualizaron "linfocitos velludos" (Tabla 1 ). En esta segunda hospitalización la concentración de IgM (monoclonal) estaba elevada 10 veces lo normal, IgA e IgG estaban reducidas y existía una elevación de la uricemia, LDH y B2 microglobulina. La ecotomografía abdomino-pelviana mostró hepatomegalia homogénea con aspecto de esteatosis y adenopatías retroperitoneales. El scanner mostró un hígado de aspecto conservado de tamaño ligeramente aumentado, riñones de aspecto normal, adenopatías en hilio hepático y en el fondo de saco de Douglas (Figuras 2 y 3 ).

Con el diagnóstico de ACLB asociada a un síndrome linfoproliferativo se indicó alopurinol, corticoides y bicarbonato de sodio endovenoso logrando aminorar la acidosis pero sin reducir las concentraciones de lactato en forma significativa (Tabla 2). Una punción medular obtuvo una muestra que se analizó con citometría de flujo. El 2\% fueron células linfoides de estirpe $\mathrm{B}$ con expresión de antígenos CD45,CD19,CD20,CD11c,CD25 y ausencia de CD10 y CD103. Las células tenían restricción en cadenas livianas con expresión de cadenas lambda. Se concluyó que existía un linfoma marginal del bazo descartándose el diagnóstico clínico previo de tricoleucemia.

A los 12 días del ingreso se decidió administrar un anticuerpo anti-CD20 (rituximab) con el fin de reducir la masa de linfocitos B de la paciente. Para ello se administró $281 \mathrm{mg}$ de rituximab (188 $\mathrm{mg} / \mathrm{m}^{2}$ ), observándose una reducción progresiva 
Tabla 1. Exámenes de laboratorio efectuados en las 2 hospitalizaciones

\begin{tabular}{|c|c|c|c|c|}
\hline & $\operatorname{1a}^{\mathrm{a}}$ & $\begin{array}{c}2^{a} \\
\text { hospitalización } \\
\text { Día de } \\
\text { admisión }\end{array}$ & $\begin{array}{l}4 \text { días post } \\
\text { Rituximab }\end{array}$ & $\begin{array}{l}\text { Después de } \\
4 \text { dosis de } \\
\text { Rituximab }\end{array}$ \\
\hline Hematocrito (\%) & 25 & 18,1 & 22,5 & 37,6 \\
\hline Hemoglobina ( $\mathrm{g} / \mathrm{dL})$ & 8,4 & 6,0 & 7,4 & 12,3 \\
\hline Leucocitos (mm3) & 5.200 & 3.800 & 3.300 & 5.100 \\
\hline Linfocitos (\%) & 78 & 50 & 23 & 3 \\
\hline Plaquetas (mm3) & 60.000 & 33.000 & 150.000 & 160.000 \\
\hline Reticulocitos (\%) & 12 & 6,7 & 6,6 & 8,0 \\
\hline Eritrobastos (\%) & 2 & 30 & 12 & 1 \\
\hline VHS (mm/hora) & 116 & $>140$ & & 26 \\
\hline B2 microglobulina $(\mathrm{mg} / \mathrm{L})(\leq 1,47)$ & 9,43 & 4,2 & & \\
\hline Protrombina (\%) (70-100) & 46,7 & 51,9 & 40,5 & 59,4 \\
\hline TTPK (seg) (20-35) & 47,3 & 58,7 & 59 & 43,8 \\
\hline Factor V lábil (\%) (60-100) & 63 & & & \\
\hline Factor VII (\%) (50-120) & 51 & & & \\
\hline Factor VIII (\%) (70-200) & 32 & & & \\
\hline Factor X (\%) (70-140) & 88,5 & & & \\
\hline Anticoagulante lúpico (seg) (20-40) & 34,1 & & & \\
\hline Ac. Anticardiolipinas IgG (GPL U/ml) (0-15) & 3,42 & & & \\
\hline Ac. Anticardiolipinas IgM (GPL U/ml) (0-15) & 28,4 & & & \\
\hline $\operatorname{lgG}(\mathrm{mg} / \mathrm{dL})(552-1631)$ & 658 & 395 & 330 & 548 \\
\hline $\lg A(\mathrm{mg} / \mathrm{dL})(70-400)$ & 62 & 61 & 480 & 340 \\
\hline $\operatorname{lgM}(\mathrm{mg} / \mathrm{dL})(40-230)$ & 1.514 & 2.379 & 1.385 & 593 \\
\hline Ferritina sérica (ng/ml) (4,63-204) & 19,76 & 297,3 & & 388 \\
\hline Ferremia (ug/dL) (25-156) & 39 & 93 & & 26 \\
\hline Saturación (\%) & 8,49 & 27,8 & & 9,3 \\
\hline PCR (mg/L) $(0,1-8,2)$ & 16,9 & 79,4 & 34,9 & 14,6 \\
\hline Creatinina $(\mathrm{mg} / \mathrm{dL})(0,6-1,1)$ & 1,25 & 1,0 & 0,70 & \\
\hline Uremia (mg/dL)(15-45) & 47,1 & 64 & & \\
\hline Glicemia (mg/dL)(65-100) & 90 & 86 & & \\
\hline Uricemia (mg/dL)(2,6-6,0) & 8,7 & 13,9 & 7,2 & 3,2 \\
\hline Calcemia (mg/dL) $(8,4-10,2)$ & 8,1 & & & 9,0 \\
\hline LDH (U/L) (125-243) & 214 & 662 & 246 & 130 \\
\hline Bilirrubina Total $(\mathrm{mg} / \mathrm{dL})(0,2-1,2)$ & 1,46 & 1,59 & & 1,46 \\
\hline Bilirrubina directa $(\mathrm{mg} / \mathrm{dL})(0,01-0,5)$ & 0,72 & 0,77 & & 0,56 \\
\hline Fosfatasas alcalinas (U/L) (40-150) & 82 & 102 & & \\
\hline GGTP (U/L) (9-36) & 12 & & & \\
\hline SGPT (U/L) (1-55) & 7 & 12 & & \\
\hline SGOT (U/L) (5-34) & 10 & 21 & & \\
\hline Colesterol (mg/dL) $(\leq 200)$ & 64 & & & \\
\hline Triglicéridos (mg/d/L) $(\leq 150)$ & 73 & & & \\
\hline Proteinemia (g/dL) $(6,4-8,3)$ & 5,9 & 7,3 & & \\
\hline Albuminemia $(\mathrm{g} / \mathrm{dL})(3,5-5,0)$ & 3,1 & 2,8 & & \\
\hline Sodio (mEq/L) (136-145) & 145 & 135 & 141 & 139 \\
\hline Potasio (mEq/L) $(3,4-4,4)$ & 3,95 & 4,45 & 3,69 & 3,59 \\
\hline Bicarbonato( mEq/L) (20-26) & 23 & 11 & 25 & 23 \\
\hline Cloro (mEq/L) (98-107) & 113 & 100 & 105 & 108 \\
\hline
\end{tabular}




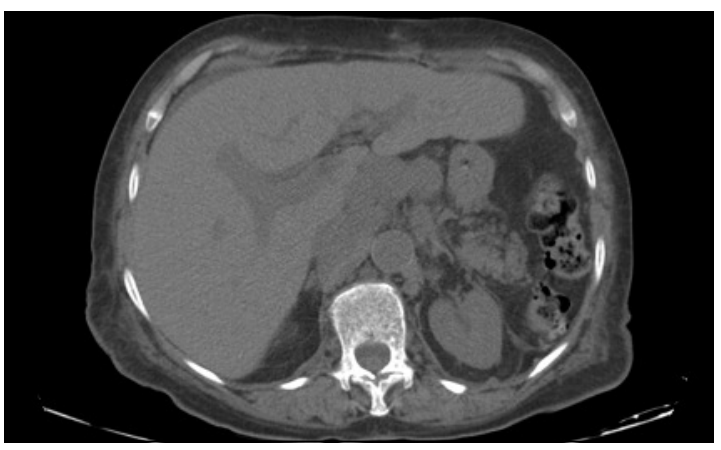

Figura 2. Imagen abdominal en tomografía computada obtenida durante la segunda hospitalización. Se observa un hígado homogéneo discretamente aumentado de tamaño con un grupo de adenopatías en su hilio. Hay ausencia del bazo (esplenectomía) y ocupación de su espacio por asas intestinales.

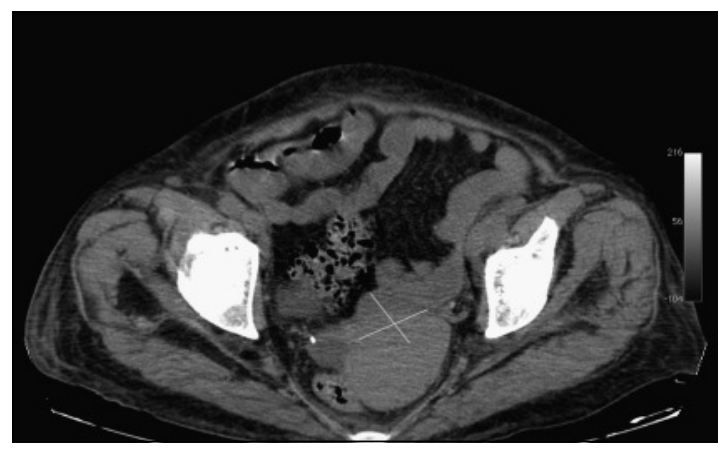

Figura 3. Imagen pelviana obtenida durante la segunda hospitalización. Se observa un conglomerado de adenopatías bajo el útero (líneas cruzadas) en la parte posterior de la pelvis.

Tabla 2. Exámenes de laboratorio y terapia durante segunda hospitalización por acidosis láctica tipo B

\begin{tabular}{|c|c|c|c|c|c|c|c|c|c|c|c|c|c|c|c|c|c|c|}
\hline $\begin{array}{l}\text { Día de hospita- } \\
\text { lización }\end{array}$ & 0 & 1 & 2 & 3 & 4 & 5 & 6 & 7 & 8 & 9 & 10 & 11 & 12 & 13 & 14 & 15 & 16 & 19 \\
\hline $\begin{array}{l}\text { Bicarbonato plas- } \\
\text { mático (mEq/L) }\end{array}$ & 11,0 & 14,5 & 19 & 23 & 21 & 21 & 21 & 20 & 20 & 22 & 24 & & 28 & & 25 & & 25 & 24 \\
\hline $\begin{array}{l}\text { Anion GAP } \\
\text { (mEq/L) } \\
(\mathrm{VN}: 12 \pm 4)\end{array}$ & 24 & & 20 & 21 & 20 & 21 & 17 & 23 & & 19 & 16 & & 13 & & 14 & & & 10 \\
\hline $\begin{array}{l}\text { Lactato (mmol/L) } \\
\text { (VN: } 0,5-2,2)\end{array}$ & & 12,2 & 10,5 & 10,6 & 11,7 & 11,9 & 10,3 & 12,1 & 12,8 & 9,3 & 8,1 & & & & 4,3 & & 2,4 & 1,6 \\
\hline $\mathrm{pH}$ & & 7,39 & 7,38 & & & & & & & & & & & & & & & \\
\hline $\mathrm{pCO}_{2}(\mathrm{~mm} / \mathrm{Hg})$ & & 24,3 & 36,7 & & & & & & & & & & & & & & & \\
\hline $\mathrm{pO}_{2}(\mathrm{~mm} / \mathrm{Hg})$ & & 111 & & & & & & & & & & & & & & & & \\
\hline $\begin{array}{l}\text { Aporte de bicarbo- } \\
\text { nato ev (mEq/día) }\end{array}$ & 24 & 240 & 240 & 80 & 80 & 80 & 80 & 80 & 80 & 80 & 80 & 80 & 80 & 60 & 60 & 60 & 40 & 0 \\
\hline $\begin{array}{l}\text { Plaquetas } \\
\left(10^{3} \times \mathrm{mm}^{3}\right)\end{array}$ & 33 & 30 & 26 & 24 & 20 & 30 & 39 & 50 & 16 & & 26 & & & 70 & 82 & & 150 & 156 \\
\hline $\begin{array}{l}\text { Tx Glóbulos rojos } \\
\text { (unidades) }\end{array}$ & 2 & 2 & & & & 2 & & & & & & & & & & & & \\
\hline Prednisona (mg) & & & & 50 & 50 & 50 & 50 & 50 & 50 & 50 & 50 & 50 & 50 & 50 & 50 & 50 & 50 & 50 \\
\hline Rituximab (mg) & & & & & & & & & & & & & 281 & & & & & \\
\hline
\end{tabular}

ev: endovenoso ; Tx: transfusión; VN: valores normales.

de los niveles de lactato hasta su normalización y un rápido incremento en el recuento de plaquetas (Tabla 2). Posteriormente, se administró la dosis usual $\left(375 \mathrm{mg} / \mathrm{m}^{2}\right)$ en las 3 semanas siguientes. Las concentraciones de lactato persistieron normales (Tabla 2) y la concentración de IgM se redujo a un $25 \%$ del valor inicial (Tabla 1).

\section{Discusión}

La ACLB se ha encontrado ocasionalmente en enfermos con enfermedades hematológicas malignas como: LNH (B,T,NK, anaplásicos de células grandes), leucemias linfoblásticas y mieloblásticas, mieloma múltiple, transformación leucémica de 
LNH y enfermedad de Hodgkin ${ }^{1,4-8}$. A junio de 2011 había 34 casos publicados de ACLB asociada a linfomas y 30 asociados a leucemias ${ }^{9,10}$. Ocasionalmente la ACLB ha sido la forma de presentación de linfomas previamente no diagnosticados y en otras, la primera manifestación de una recaída después de una quimioterapia exitosa o de un trasplante de médula ósea ${ }^{11}$. La ACLB frecuentemente se asocia a hipoglicemia ${ }^{5,11-14}$.

El LNH marginal esplénico es una variedad poco frecuente que representa menos del $2 \%$ de los linfomas, $2 / 3$ presentan prolongaciones citoplasmáticas en los linfocitos de sangre periférica. Inmunofenotípicamente expresan CD19,CD20,CD22 y PAX5 y no expresan CD25 y CD103, lo que ayuda a diferenciarlo de la tricoleucemia ${ }^{15,16}$. En esta paciente, si se hubiera podido contar con la determinación del inmunofenotipo en la primera hospitalización, se habría conocido el tipo específico de linfoma que padecía, lo que hubiera permitido haber iniciado su terapia un año antes, evitando la complicación que motivó la comunicación de este caso. Desgraciadamente su familia no aceptó asumir el elevado gasto que dicho examen ameritaba.

El mecanismo de producción de la ACLB en estas enfermedades no está claramente comprendido $^{1,10}$. Se han planteado diversas hipótesis como: alto consumo de glucosa y producción excesiva de ácido láctico por las células tumorales mediante glicolisis anaerobia, la que sobrepasaría el clearance hepático ${ }^{10,14,17,18}$; infiltración hepática y/o renal por células tumorales ${ }^{10}$; deficiencia de tiamina o riboflavina, particularmente en pacientes que están con nutrición parenteral exclusiva o utilizan metotrexato, por un trastorno en el complejo enzimático piruvato-deshidrogenasa ${ }^{4,19}$; glicolisis aumentada por sobreexpresión o expresión aberrante de enzimas glicolíticas en las células tumorales como hexoquinasas mediadas por IGF-1 o sus receptores y disfunción mitocondrial; e isquemia tisular debido a micro-émbolos de células neoplásicas con obstrucción de lechos vasculares $^{4,20}$. Mediante el uso del PET-Scan se ha demostrado la existencia de un aumento del metabolismo de la glucosa en los sitios tumorales mediante el empleo de 2-fluro-2-deoxy-D-glucosa como trazador ${ }^{21}$. Aproximadamente, $90 \%$ de los pacientes tienen infiltración hepática por células tumorales ${ }^{10,22}$. Se han encontrado niveles elevados de IGF-1, proteína trasportadora de IGF-1 y factor de necrosis tumoral alfa (TNF) asociado a la ACLB, aunque su rol en la génesis no se ha esclarecido ${ }^{4,10}$. Los niveles elevados de TNF causan una reducción en la piruvato-deshidrogenasa e inhiben el sistema de transporte de electrones, lo que resulta en aumento de los niveles de lactato. Ellos se normalizan después de reducirse los niveles de lactato a lo normal ${ }^{4,21}$.

El clearance de lactato aproximadamente en $90 \%$ es hepático y $10 \%$ renal $^{4}$. Se han comunicado algunos casos de ACLB asociada a linfomas con compromiso tumoral hepático en pacientes con infecciones por VIH y virus $C$ de la hepatitis ${ }^{17,23} y$ también por infiltración de los riñones por células tumorales en linfomas y leucemias ${ }^{20,24}$.

Se ha recomendado sospechar la presencia de un linfoma frente a ACL sin que exista hipoxia especialmente cuando se asocia a hipoglicemia y también en los casos de insuficiencia hepática fulminante sin etiología conocida ${ }^{4,20,25}$.

La ACLB asociada a linfomas tiene un mal pronóstico, más de $75 \%$ de los pacientes mueren dentro del mes de su presentación clínica y $92 \%$ durante el seguimiento ${ }^{5,9,22}$.

El bicarbonato endovenoso se utiliza como medida transitoria para mitigar los efectos de la acidosis, particularmente la fatiga respiratoria e inestabilidad hemodinámica. Sin embargo, su utilidad es limitada para corregir la acidosis y puede aumentar la generación de lactato ${ }^{13}$. En esta enferma, si bien normalizó los niveles de bicarbonato e hizo desaparecer la polipnea, no modificó significativamente los niveles de lactato. Ocasionalmente se ha usado diálisis para remover el exceso de lactato y estabilizar al paciente previo al inicio de la quimioterapia, ${ }^{9,13}$. La quimioterapia sola o acompañada de rituximab o radioterapia, han sido la única forma de corregir la $\mathrm{ACLB}^{9,10,20,26}$. En esta paciente una sola dosis de rituximab (50\% de la habitual) permitió suspender la administración de bicarbonato, corregir la acidosis y superar la trombocitopenia. Probablemente la destrucción masiva de células B redujo la generación excesiva de lactato. De las teorías disponibles para explicar la ACLB, en esta paciente pueden ser descartadas la infiltración parenquimatosa hepática y renal por células neoplásicas, dada la ausencia de alteraciones morfológicas y funcionales de estos órganos. La presencia de inhibidores de la coagulación y de un componente $\mathrm{M}$ en esta paciente son fenómenos asociados a LNH de estirpe B. También, la 
presencia de anticuerpos anticardiolipinas ha sido descrita en pacientes con linfomas marginales ${ }^{27}$. Las masas en hilio hepático y en la pelvis probablemente corresponden a adenopatías.

Este caso clínico es un buen ejemplo para recalcar que en el tratamiento de una acidosis metabólica es fundamental abordar directamente su etiología.

La edad media de presentación de los LNH marginales de bazo es 68 años. Suelen debutar con esplenomegalia sintomática, a veces acompañada de linfocitosis, $80 \%$ de los pacientes tienen infiltración de la médula ósea, $30 \%$ hepática y $25 \%$ adenopatías retroperitoneales. La esplenectomía es la terapia de elección ya que corrige las citopenias, alivia los síntomas y cuando ello no es suficiente se ha usado quimioterapia (fludarabina, pentostatin). En ancianos o pacientes con falla renal se ha utilizado rituximab con buenos resultados ${ }^{15,16,28,29}$.

En suma: comunicamos el caso de una paciente anciana con un linfoma marginal del bazo que desarrolló una ACLB manifestada por una intensa polipnea, 12 meses después del diagnóstico de un LNH. El uso de rituximab corrigió rápidamente el trastorno. Sugerimos descartar un proceso maligno hematológico frente a una ACL inexplicada.

\section{Referencias}

1. Chang H, Shuai X, Ma HB, Liu T. A case of acute lymphoblastic leukemia complicated by lactic acidosis. Int J Hematol 2010; 92: 538-41.

2. Madias NE. Lactic acidosis. Kidney Int 1986; 29: 752-74.

3. Kreisberg RA. Lactate homeostasis and lactic acidosis. Ann Intern Med 1980; 92: 227-37.

4. Friedenberg AS, Brandoff DE, Schiffman FJ. Type B lactic acidosis as a severe metabolic complication in lymphoma and leukemia: a case series from a single institution and a literature review. Medicine (Baltimore) 2007; 86: 225-32.

5. He YF, Wei W, Sun ZM, Ji CS, Wang G, Chen MP, et al. Fatal lactic acidosis and hypoglycemia in a patient with relapsed natural killer/T-cell lymphoma. Adv Ther 2007; 24: 505-9.

6. Dogan E, Erkoc R, Sayarlioglu H, Alici S, Dilek I, Alici O. Fatal lactic acidosis due to leukemic transformation in a patient with non-Hodgkin's lymphoma:case report. Adv Ther 2005; 22: 4436.

7. Ustun C, Fall P, Szerlip HM, Jillella A, Hendricks L, Burgess $\mathrm{R}$ et al. Multiple myeloma associated with lactic acidosis. Leuk Lymphoma 2002; 43: 2395-7.
8. Thakur V, Sander G, Rab ST. Hodgkin's disease and lactic acidosis. Nephron 2001; 88: 276-7.

9. Chan FH, Carl D, Lyckholm LJ. Severe lactic acidosis in a patient with B-cell lymphoma: a case report and review of the literature. Case Report Med 2009; 2009: 534-61.

10. Sillos EM, Shenep JL, Burghen GA, Pul CH, Behm FG, Sandlund JT. Lactic acidosis: a metabolic complication of hematologic malignancies: case report and review of the literature. Cancer 2001; 92: 2237-46.

11. Luscri N, Mauer M, Sarafoglou K, Moran A, Tolar J. Lactic acidosis and hypoglycemia with ALL relapse following engrafted bone marrow transplant. Pediatr Blood Cancer 2009; 53: 223-5.

12. Díaz J, Antoine J, Azad N. A case of hypoglycemia, lactic acidosis, and hematologic malignancy. Endocr Pract. 2010; 16: 241-3.

13. Prikis M, Bhasin V, Young MP, Gennari FJ, Rimmer JM. Sustained low-efficiency dialysis as a treatment modality in a patient with lymphoma-associated lactic acidosis. Nephrol Dial Transplant 2007; 22: 2383-5.

14. Abrahamian H, Pecherstorfer M, Vesely M, Baumgartner G, Irsigler K. Recurrent hypoglycemia and lactate acidosis in non-Hodgkin's lymphoma. Dtsch Med Wochenschr 1991; 116: 1428-32.

15. Khal B, Yang D. Marginal zone lymphoma: management of nodal, splenic and MALT NHL. Hematology Am Soc Hematol Educ Program 2008: 359-64.

16. Zucca E, Bertoni F, Stathis A, Cavalli F. Marginal zone lymphomas. Hematol Oncol Clin N Am 2008; 22: 883901.

17. Kestler MH, Gardner EM, Cohn DL. Hepatic nonHodgkin's lymphoma with lactic acidosis in HIV-infected patients: report of 2 cases. J Int Assoc Physicians AIDS Care (Chic) 2010; 9: 301-5.

18. Mosunjak MB, Sundstrom JB, Mosunjac MI. Unusual presentation of anaplastic large cell lymphoma with clinical course mimicking fever of unknown origin and sepsis: autopsy study of five cases. Croat Med J 2008; 49: 660-8.

19. Svahn J, Schiaffino MC, Caruso U, Calvillo M, Minniti G, Dufour C. Severe lactic acidosis due to thiamine deficiency in a patient with B-cell leukemia/lymphoma on total parenteral nutrition during high-dose methotrexate therapy. J Pediatr Hematol Oncol 2003; 25: 965-8.

20. De Keulenaer B, Van Outryve S, De Baker A, Van Overbeke L, Daelemans R, Van Marck E et al. Symptomatic lactic acidosis due to relapse of T-cell acute lymphoblastic leukaemia in the kidney. Nephrol Dial Transplant 2003; 18: 1214-6.

21. Durig J, Fiedler W, de Wit M, Steffen M, Hossfeld DK. 
Lactic acidosis and hypoglycemia in a patient with high-grade non-Hodgkin's lymphoma and elevated circulating TNF-alpha. Ann Hematol 1996; 72: 97-9.

22. Glasheen JJ, Sorensen MD. Burkitt's lymphoma presenting with lactic acidosis and hypoglycemia-a case presentation. Leuk Lymphoma 2005; 46: 281-3.

23. Cofré P, Valera JM, Smok G, Regonessi C, Brahm J. Fulminant liver failure associated with T-cell nonHodgkin's lymphoma and hepatitis $\mathrm{C}$ virus. A case report.Gastroenterol Hepatol 2006; 29: 542-5.

24. Révész T, Obeid K, Mpofu C. Severe lactic acidosis and renal involvement in a patient with relapsed Burkitt's lymphoma. Pediatr Hematol Oncol 1995; 12: 283-8.

25. Padilla GF, Garibay MA, Hummel HN, Avila R, Méndez A, Ramírez R. [Fulminant non-Hodgkin lymphoma presenting as lactic acidosis and acute liver failure: case report and literature review]. Acta Gastroenterol Lati- noam 2009; 39: 129-34.

26. Ohtsubo K, Imamura R, Seki R, Ohshima K, Hashiguchi $\mathrm{M}$, Yakashiji $\mathrm{K}$ et al. Blastoid variant of mantle cell lymphoma with lactic acidosis:a case report. Int J Hematol 2004; 80: 428-31.

27. Harner KC, Jackson LW, Drabick JJ. Normalization of anticardiolipin antibodies following Rituximab therapy for marginal zone lymphoma in a patient with Sjögren syndrome. Rheumatology 2004; 43: 1309-10.

28. Bennett M, Yegena S, Dave HP, Schechter GP. Rituximab monotherapy is highly effective in splenic marginal zone lymphoma. Hematol Oncol 2008; 26: 114.

29. Kalpadakis C, Pangalis GA, Dimopoulos MN, Vassilakopoulos TP, Kyrtsonis MC, Korkolopoulou P, et al. Rituximab monotherapy is highly effective in splenic marginal zone lymphoma. Hematol Oncol 2007; 25: 127-31. 